\title{
Edge-Neighbor-Rupture Degree of Graphs
}

\author{
Ersin Aslan \\ Turgutlu Vocational Training School, Celal Bayar University, Turgutlu, 45400 Manisa, Turkey
}

Correspondence should be addressed to Ersin Aslan; ersin.aslan@cbu.edu.tr

Received 22 April 2013; Accepted 3 August 2013

Academic Editor: Frank Werner

Copyright (C) 2013 Ersin Aslan. This is an open access article distributed under the Creative Commons Attribution License, which permits unrestricted use, distribution, and reproduction in any medium, provided the original work is properly cited.

The edge-neighbor-rupture degree of a connected graph $G$ is defined to be $\operatorname{ENR}(G)=\max \{\omega(G-S)-|S|-m(G-S): S \subseteq E(G)$, $\omega(G-S) \geq 1\}$, where $S$ is any edge-cut-strategy of $G, \omega(G-S)$ is the number of the components of $G-S$, and $m(G-S)$ is the maximum order of the components of $G-S$. In this paper, the edge-neighbor-rupture degree of some graphs is obtained and the relations between edge-neighbor-rupture degree and other parameters are determined.

\section{Introduction}

In a communication network, the vulnerability measures the resistance of the network to disruption of operation after the failure of certain stations or communication links. To measure the vulnerability we have some parameters which are connectivity [1], integrity [2], scattering number [3], and rupture degree [4].

A spy network can be modeled by a graph whose vertices represent the stations and whose edges represent the lines of communication. If a station is destroyed, the adjacent stations will be betrayed so that the betrayed stations become useless to network as a whole [5]. Therefore, instead of considering the stability of a communication network in standard sense, some new graph parameters such as vertex-neighbor-connectivity [6] and edge-neighborconnectivity [7], vertex-neighbor-integrity [8] and edgeneighbor-integrity [9], vertex-neighbor-scattering number [10] and edge-neighbor-scattering number [11], and vertexneighbor-rupture degree [12] were introduced to measure the stability of communication networks in "neighbor" sense.

We use Bondy and Murty [1] for terminology and notation not defined here and consider only finite simple connected graphs. Let $G=(V, E)$ be a graph and $e$ any edge in $G$. The diameter of $G$, denoted by $\operatorname{diam}(G)$, is the maximum distance over all pairs of vertices in $G$.

$N(e)=\{f \in E(G) \mid f \neq e ; e$ and $f$ are adjacent $\}$ is the open-edge-neighborhood of $e$, and $N[e]=N(e) \cup\{e\}$ is the closed-edge-neighborhood of $e$. An edge $e$ in $G$ is said to be subverted when $N[e]$ is deleted from $G$. In other words, if $e=$ $[u, v], G-N[e]=G-\{u, v\}$. A set of edges $S$ is called an edge subversion strategy of $G$ if each of the edges in $S$ has been subverted from $G$. The survival subgraph is denoted by $G-S$. An edge subversion strategy $S$ is called an edge-cut-strategy of $G$ if the survival subgraph $G-S$ is disconnected or is a single vertex or the empty graph [13].

The edge-neighbor-connectivity of $G, \Lambda(G)$, is the minimum size of all edge-cut-strategies of $G$. A graph $G$ is m-edgeneighbor-connected if $\Lambda(G)=m$ [7].

The edge-neighbor-integrity of a graph $G, \operatorname{ENI}(G)$, is defined to be

$$
\operatorname{ENI}(G)=\min _{S \subseteq E(G)}\{|S|+m(G-S)\}
$$

where $S$ is any edge subversion strategy of $G$ and $m(G-S)$ is maximum order of the components of $G-S$ [9].

The edge-neighbor-scattering number of $G, \operatorname{ENS}(G)$, is defined as

$$
\operatorname{ENS}(G)=\max _{S \subseteq E(G)}\{\omega(G-S)-|S|\}
$$

where $S$ is any edge-cut-strategy of $G$ and $\omega(G-S)$ is the number of the components of $G-S$ [11].

The known parameters concerning the neighborhoods do not deal with the number of the removing edges, the number of the components, and the number of the vertices in the largest component of the remaining graph in a disrupted network simultaneously. In order to fill this void 


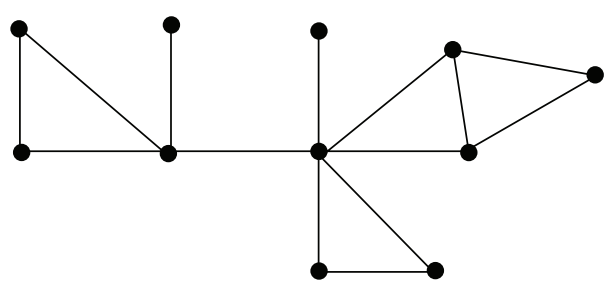

$G_{1}$

(a)

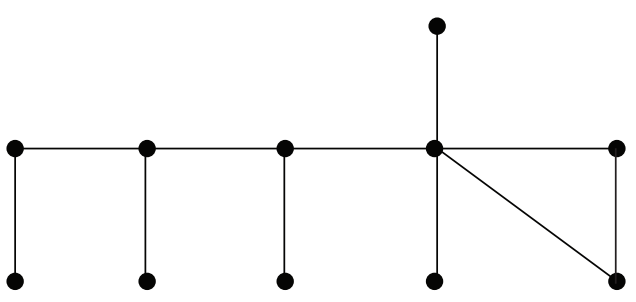

$G_{2}$

(b)

FIgURE 1: The graphs $G_{1}$ and $G_{2}$.

in the literature, the current study proposes a definition of edge-neighbor-rupture degree which is a new parameter concerning these three values. Additionally, this study also analyzes the relations between edge-neighbor-rupture degree and some other parameters and obtains edge-neighborrupture degree of some graphs.

The edge-neighbor-rupture degree of a connected graph $G$ is defined to be

$$
\begin{gathered}
\operatorname{ENR}(G)=\max \{\omega(G-S)-|S|-m(G-S): S \subseteq E(G), \\
\omega(G-S) \geq 1\},
\end{gathered}
$$

where $S$ is any edge-cut-strategy of $G, \omega(G-S)$ is the number of the components of $G-S$, and $m(G-S)$ is the maximum order of the components of $G-S$. A set $S^{*} \subseteq E(G)$ is said to be the ENR-set of $G$ if $\operatorname{ENR}(G)=\omega\left(G-S^{*}\right)-\left|S^{*}\right|-m\left(G-S^{*}\right)$.

The edge-neighbor-rupture degree differs from edgeneighbor-connectivity, edge-neighbor-integrity, and edgeneighbor-scattering number in showing the vulnerability of networks. For example, consider the graphs $G_{1}$ and $G_{2}$ in Figure 1.

It can be easily seen that the edge-neighbor-connectivity, edge-neighbor-integrity, and edge-neighbor-scattering number of these graphs are equal:

$$
\begin{aligned}
\Lambda\left(G_{1}\right) & =\Lambda\left(G_{2}\right)=1, \\
\operatorname{ENI}\left(G_{1}\right) & =\operatorname{ENI}\left(G_{2}\right)=4, \\
\operatorname{ENS}\left(G_{1}\right) & =\operatorname{ENS}\left(G_{2}\right)=4 .
\end{aligned}
$$

On the other hand, the edge-neighbor-rupture degrees of $G_{1}$ and $G_{2}$ are different:

$$
\begin{aligned}
& \operatorname{ENR}\left(G_{1}\right)=1, \\
& \operatorname{ENR}\left(G_{2}\right)=2 .
\end{aligned}
$$

Hence, the edge-neighbor-rupture degree is a better parameter for distinguishing vulnerability of graphs $G_{1}$ and $G_{2}$.

\section{Bounds for Edge-Neighbor-Rupture Degree}

In this section some lower and upper bounds are given for the edge-neighbor-rupture degree of a graph using different graph parameters.

Theorem 1. Let $G$ be a connected graph of order $n$. Then,

$$
\operatorname{ENR}(G) \leq n-4 .
$$

Proof. Let $S$ be an edge-cut-strategy of $G$ and $|S|=r$. If $r \geq 1$, then $\omega(G-S) \leq n-2$ and $m(G-S) \geq 1$. Therefore,

$$
\omega(G-S)-|S|-m(G-S) \leq n-2-1-1 .
$$

Hence we have

$$
\operatorname{ENR}(G) \leq n-4 .
$$

The proof is completed.

Theorem 2. Let $G$ be a connected graph of order $n$, and let $\alpha(G), \Lambda(G)$ be the independent number and edge-neighborconnectivity of $G$, respectively. Then,

$$
\operatorname{ENR}(G) \leq \alpha(G)-\Lambda(G)-1 .
$$

Proof. Let $S$ be an edge-cut-strategy of $G$. For any $S$ of $G,|S| \geq$ $\Lambda(G), \omega(G-S) \leq \alpha(G)$, and $m(G-S) \geq 1$. Hence we get

$$
\operatorname{ENR}(G) \leq \alpha(G)-\Lambda(G)-1 .
$$

The proof is completed.

Theorem 3. Let $G$ be a connected graph of order $n \geq 3$. If $\operatorname{ENR}(G)=n-4$; then $\operatorname{diam}(G) \leq 3$.

Proof. Assume that $\operatorname{diam}(G) \geq 4$; then $G$ contains a path $P_{5}$. Thus for any edge $e$ in $G, \omega(G-S) \leq n-2, m(G-\{e\}) \geq$ 2 , and for any two edges $e_{1}$ and $e_{2}$ in $G, \omega(G-e) \leq n-2$, $m\left(G-\left\{e_{l}, e_{2}\right\}\right) \geq 1$. Therefore

$$
\operatorname{ENR}(G) \leq n-5,
$$

a contradiction. Hence $\operatorname{diam}(G) \leq 3$.

The proof is completed. 
Theorem 4. Let $G$ be a connected graph of order $n$ and $\alpha^{\prime}(G)$ edge independence number of $G$. Then,

$$
\operatorname{ENR}(G) \leq n-3 \alpha^{\prime}(G)-1 .
$$

Proof. Let $S$ be an edge-cut-strategy of $G$. If $|S|=\alpha^{\prime}(G)$, then $G-S$ contains $n-2 \alpha^{\prime}(G)$ isolated vertices and $m(G-S) \geq 1$. From the definition of edge neighbor rupture degree we have

$$
\operatorname{ENR}(G) \leq n-3 \alpha^{\prime}(G)-1 .
$$

The proof is completed.

\section{Edge-Neighbor-Rupture Degree of Some Graphs}

In this section, we consider the edge-neighbor-rupture degree of some graphs.

Theorem 5. Let $P_{n}$ be a path with order $n(\geq 4)$. Then

$$
\operatorname{ENR}\left(P_{n}\right)= \begin{cases}0, & n \equiv 1(\bmod 3), \\ -1, & n \equiv 0,2(\bmod 3) .\end{cases}
$$

Proof. Let $S$ be an edge-cut-strategy of $P_{n}$ and $|S|=r$. If $r \leq$ $\lfloor(n-1) / 3\rfloor$, then $\omega\left(P_{n}-S\right) \leq r+1$ and $m\left(P_{n}-S\right) \geq\lceil(n-$ $2 r) /(r+1)\rceil$. Thus

$$
\begin{gathered}
\omega\left(P_{n}-S\right)-|S|-m\left(P_{n}-S\right) \leq r+1-r-\left\lceil\frac{n-2 r}{r+1}\right\rceil \\
\operatorname{ENR}\left(P_{n}\right) \leq \max _{r}\left\{1-\left\lceil\frac{n-2 r}{r+1}\right\rceil\right\},
\end{gathered}
$$

the function $f(r)$ takes its maximum value at $r=\lfloor(n-1) / 3\rfloor$, and we get $\operatorname{ENR}\left(P_{n}\right) \leq-1$ where $n \equiv 0,2(\operatorname{Mod} 3)$ and $\operatorname{ENR}\left(P_{n}\right) \leq 0$ where $n \equiv 1(\operatorname{Mod} 3)$. So, we have

$$
\operatorname{ENR}\left(P_{n}\right) \leq \begin{cases}0, & n \equiv 1(\bmod 3) \\ -1, & n \equiv 0,2(\bmod 3) .\end{cases}
$$

On the other hand, if $r \geq\lfloor(n-1) / 3\rfloor+1$, then we have $\omega\left(P_{n}-S\right) \leq r$ and $m\left(P_{n}-S\right) \geq 1$. Hence

$$
\begin{gathered}
\omega\left(P_{n}-S\right)-|S|-m\left(P_{n}-S\right) \leq r-r-1, \\
\operatorname{ENR}\left(P_{n}\right) \leq-1 .
\end{gathered}
$$

It can be easily seen that there is an edge-cut-strategy $S^{*}$ of $P_{n}$ such that $\left|S^{*}\right|=\lfloor(n-1) / 3\rfloor, \omega\left(P_{n}-S^{*}\right)=\lfloor(n-1) / 3\rfloor+1$, and $m\left(P_{n}-S^{*}\right)=2$ where $n \equiv 0,2(\operatorname{Mod} 3)$ and $m\left(P_{n}-S^{*}\right)=1$ where $n \equiv 1(\operatorname{Mod} 3)$. Therefore,

$$
\operatorname{ENR}\left(P_{n}\right)= \begin{cases}0, & n \equiv 1(\bmod 3), \\ -1, & n \equiv 0,2(\bmod 3) .\end{cases}
$$

The proof is completed by (16), (17), and (18).

Theorem 6. Let $C_{n}$ be a cycle with order $n(\geq 6)$. Then

$$
\operatorname{ENR}\left(C_{n}\right)= \begin{cases}-1, & n \equiv 0(\bmod 3), \\ -2, & n \equiv 1,2(\bmod 3) .\end{cases}
$$

Proof. Let $S$ be an edge-cut-strategy of $C_{n}$ and $|S|=r$. If $r \leq$ $\lfloor n / 3\rfloor$, then $\omega\left(C_{n}-S\right) \leq r$ and $m\left(C_{n}-S\right) \geq\lceil(n-2 r) / r\rceil$. Thus

$$
\begin{gathered}
\omega\left(C_{n}-S\right)-|S|-m\left(C_{n}-S\right) \leq r-r-\left\lceil\frac{n-2 r}{r}\right\rceil, \\
\operatorname{ENR}\left(C_{n}\right) \leq \max _{r}\left\{\left\lceil\frac{n-2 r}{r}\right\rceil\right\},
\end{gathered}
$$

the function $f(r)$ takes its maximum value at $r=\lfloor n / 3\rfloor$, and we get $\operatorname{ENR}\left(C_{n}\right) \leq-1$ where $n \equiv 0(\operatorname{Mod} 3)$ and $\operatorname{ENR}\left(C_{n}\right) \leq$ -2 where $n \equiv 1,2(\operatorname{Mod} 3)$. So, we have

$$
\operatorname{ENR}\left(C_{n}\right) \leq \begin{cases}-1, & n \equiv 0(\bmod 3), \\ -2, & n \equiv 1,2(\bmod 3) .\end{cases}
$$

On the other hand, if $r \geq\lfloor n / 3\rfloor+1$, then we have $\omega\left(C_{n}-\right.$ $S) \leq r-1$ and $m\left(C_{n}-S\right) \geq 1$. Hence

$$
\begin{gathered}
\omega\left(C_{n}-S\right)-|S|-m\left(C_{n}-S\right) \leq r-1-r-1 \\
\operatorname{ENR}\left(C_{n}\right) \leq-2 .
\end{gathered}
$$

It can be easily seen that there is an edge-cut-strategy $S^{*}$ of $C_{n}$ such that $\left|S^{*}\right|=\lfloor n / 3\rfloor, \omega\left(C_{n}-S^{*}\right)=\lfloor n / 3\rfloor$, and $m\left(C_{n}-\right.$ $\left.S^{*}\right)=2$ where $n \equiv 1,2(\operatorname{Mod} 3)$ and $m\left(C_{n}-S^{*}\right)=1$ where $n \equiv 0(\operatorname{Mod} 3)$. Therefore

$$
\operatorname{ENR}\left(C_{n}\right)= \begin{cases}-1, & n \equiv 0(\bmod 3), \\ -2, & n \equiv 1,2(\bmod 3) .\end{cases}
$$

The proof is completed by (21), (22), and (23).

Lemma 7 (see [7]). For any graph $G$ with order $n, \Lambda(G) \leq$ $\lfloor n / 2\rfloor$.

Theorem 8. Let $K_{n}$ be a complete graph with order $n$. Then

$$
\operatorname{ENR}\left(K_{n}\right)=-\left\lfloor\frac{n}{2}\right\rfloor .
$$

Proof. Let $S$ be an edge-cut-strategy of $K_{n}$ and $|S|=r$. By Lemma 7 we know $\Lambda\left(K_{n}\right)=\lfloor n / 2\rfloor$. If $r \geq\lfloor n / 2\rfloor$, then $\omega\left(K_{n}-\right.$ $S) \leq 1$ and $m\left(K_{n}-S\right) \geq 1$. Hence,

$$
\begin{gathered}
\omega\left(K_{n}-S\right)-|S|-m\left(K_{n}-S\right) \leq 1-\left\lfloor\frac{n}{2}\right\rfloor-1, \\
\operatorname{ENR}\left(K_{n}\right) \leq-\left\lfloor\frac{n}{2}\right\rfloor .
\end{gathered}
$$

It can be easily seen that there is an edge set $S^{*}$ of $K_{n}$ such that $\left|S^{*}\right|=\lfloor n / 2\rfloor$, then we have $\omega\left(K_{n}-S\right)=1$ and $m\left(K_{n}-S\right)=$ 1 . From the definition of edge-neighbor-rupture degree we have

$$
\operatorname{ENR}\left(K_{n}\right)=-\left\lfloor\frac{n}{2}\right\rfloor .
$$

The proof is completed.

Definition 9. We also call $K_{1, n}$ a star with $n+1$ vertices. Let $\operatorname{DS}\left(n_{l}, n_{2}\right)$ be a double star with $\left\{n_{l}, n_{2}\right\}$ end-vertices, where $n_{1} \geq 0$ and $n_{2} \geq 0$, and a common edge [u,v], as shown in Figure 2. Note that if either $n_{1}$ or $n_{2}$ is 0 , then the double star $\operatorname{DS}\left(n_{l}, n_{2}\right)$ is a star. 


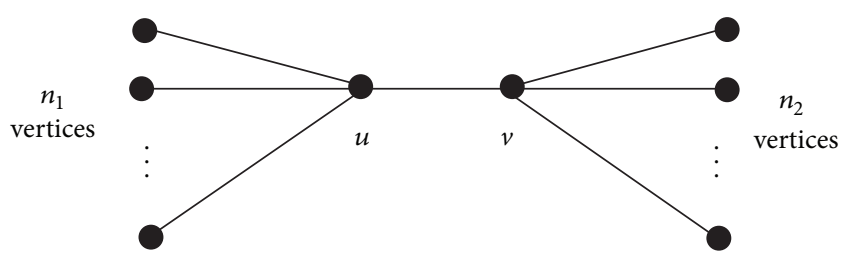

FIGURE 2: DS $\left(n_{l}, n_{2}\right)$.

Theorem 10. Let $T$ be a tree of order $n \geq 4$. Then $\operatorname{ENR}(T)=$ $n-4$ if and only if $T$ is either a star $K_{1, n-1}$ or a double star $\operatorname{DS}\left(n_{l}, n_{2}\right)$, where $n_{1} \geq 1, n_{2} \geq 1$, and $n_{1}+n_{2}=n-2$.

Proof. If $T$ is a tree of order $n \geq 3$ and $\operatorname{ENR}(T)=n-4$, then by Theorem 3 we have either $\operatorname{diam}(T)=2$ or $\operatorname{diam}(T)=3$. If $\operatorname{diam}(T)=2$, then $T$ is a $\operatorname{star} K_{1, n-1}$. If $\operatorname{diam}(T)=3$, then $T$ is a double star DS $\left(n_{l}, n_{2}\right)$, where $n_{1}>0, n_{2}>0$, and $n_{1}+n_{2}=$ $n-2$.

Conversely, let $T$ be either a star $K_{1, n-1}$ with the order $n \geq$ 4 or a double star DS $\left(n_{l}, n_{2}\right)$, where $n_{1} \geq 1, n_{2} \geq 1$, and $n_{1}+$ $n_{2}+2=n \geq 4$.

If $S$ is an edge-cut-strategy of $K_{1, n-1}$ and $|S|=1$, then we have $n-2$ isolated vertices. Therefore we have $\omega(T-S)=n-2$ and $m(T-S)=1$. So

$$
\begin{gathered}
\omega\left(K_{1, n-1}-S\right)-|S|-m\left(K_{1, n-1}-S\right)=n-2-1-1 \\
\operatorname{ENR}\left(K_{1, n-1}\right)=n-4 .
\end{gathered}
$$

It can be easily seen that there is an edge-cut-strategy $S^{*}$ of $\operatorname{DS}\left(n_{l}, n_{2}\right)$ such that $\left|S^{*}\right|=1$; then we have $\omega\left(\operatorname{DS}\left(n_{l}, n_{2}\right)-\right.$ $\left.S^{*}\right)=n-2$ and $m\left(\operatorname{DS}\left(n_{l}, n_{2}\right)-S^{*}\right)=1$. So

$$
\begin{gathered}
\omega\left(\mathrm{DS}\left(n_{l}, n_{2}\right)-S^{*}\right)-\left|S^{*}\right|-m\left(\operatorname{DS}\left(n_{l}, n_{2}\right)-S^{*}\right) \\
=n-2-1-1 \\
\operatorname{ENR}\left(\operatorname{DS}\left(n_{l}, n_{2}\right)\right)=n-4 .
\end{gathered}
$$

If $S$ is an edge set of $\operatorname{DS}\left(n_{l}, n_{2}\right)$ and $|S|>1$, then we have $\omega\left(\operatorname{DS}\left(n_{l}, n_{2}\right)-S\right)=1$ and $m\left(\operatorname{DS}\left(n_{l}, n_{2}\right)-S\right) \geq 2$.

The proof is completed.

Theorem 11. Let $K_{m, n}$ be a complete bipartite graph with $\mid m-$ $n \mid \geq 1$. Then

$$
\operatorname{ENR}\left(K_{m, n}\right)= \begin{cases}m-2 n-1, & \text { if } m>n, \\ n-2 m-1, & \text { if } m<n .\end{cases}
$$

Proof. Assume $m>n$. Let $S$ be an edge-cut-strategy of $K_{m, n}$ and $|S|=r$. If $r \geq n$, then $\omega\left(K_{m, n}-S\right) \leq m-n$ and $m\left(K_{m, n}-\right.$ $S) \geq 1$. Hence,

$$
\omega\left(K_{m, n}-S\right)-|S|-m\left(K_{m, n}-S\right) \leq m-n-r-1,
$$

the function $f(r)$ is a decreasing function and takes its maximum value at $r=n$, and we get

$$
\operatorname{ENR}\left(K_{m, n}\right) \leq m-2 n-1 .
$$

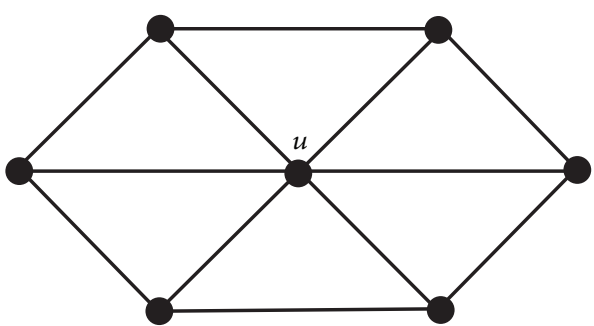

Figure 3: The wheel graph $W_{6}$.

It can be easily seen that there is an edge set $S^{*}$ of $K_{m, n}$ such that $\left|S^{*}\right|=n$; then we have $\omega\left(K_{m, n}-S\right)=m-n$ and $m\left(K_{m, n}-S\right)=1$. From the definition of edge neighbor rupture degree we have

$$
\operatorname{ENR}\left(K_{m, n}\right)=m-2 n-1 .
$$

Similarly, we obtain $\operatorname{ENR}\left(K_{m, n}\right)=n-2 m-1$ when $n>m$. Finally, we have

$$
\operatorname{ENR}\left(K_{m, n}\right)= \begin{cases}m-2 n-1, & \text { if } m>n, \\ n-2 m-1, & \text { if } m<n .\end{cases}
$$

The proof is completed.

Definition 12. The wheel graph with $n$ spokes, $W_{n}$, is the graph that consists of an $n$-cycle and one additional vertex, say $u$, that is adjacent to all the vertices of the cycle. In Figure 3, we display $W_{6}$.

Theorem 13. Let $W_{n}$ be a wheel graph with order $n(\geq 5)$. Then

$$
\operatorname{ENR}\left(W_{n}\right)= \begin{cases}-1, & n \equiv 2(\bmod 3), \\ -2, & n \equiv 0,1(\bmod 3) .\end{cases}
$$

Proof. The graph $W_{n}$ has subgraphs $C_{n}$ and $K_{1, n}$. Let $e$ be any one edge of $K_{1, n}$.

If $e \in S$ and $|S|=1$, then we get $\omega\left(W_{n}-S\right)=P_{n-1}$. So,

$$
\operatorname{ENR}\left(W_{n}\right)=\operatorname{ENR}\left(P_{n-1}\right)-1 .
$$
Hence,

If $e \notin S$ and $|S|=1$, then $\omega\left(W_{n}-S\right)=1$ and $m\left(W_{n}-S\right) \geq 2$.

$$
\operatorname{ENR}\left(W_{n}\right) \leq \omega\left(W_{n}-S\right)-|S|-m\left(W_{n}-S\right) \leq 1-1-2=-2 .
$$
Thus,

If $e \notin S$ and $|S| \geq 2$, then $\omega\left(W_{n}-S\right)=1$ and $m\left(W_{n}-S\right) \geq 1$.

$\operatorname{ENR}\left(W_{n}\right) \leq \omega\left(W_{n}-S\right)-|S|-m\left(W_{n}-S\right) \leq 1-2-1=-2$.

The proof is completed by (35), (36), and (37).

\section{References}

[1] J. A. Bondy and U. S. R. Murty, Graph Theory with Applications, The Macmillan Press, 1976. 
[2] C. A. Barefoot, R. Entringer, and H. Swart, "Vulnerability in graphs-a comparative survey," Journal of Combinatorial Mathematics and Combinatorial Computing, vol. 1, pp. 13-22, 1987.

[3] H. A. Jung, "On a class of posets and the corresponding comparability graphs," Journal of Combinatorial Theory B, vol. 24, no. 2, pp. 125-133, 1978.

[4] Y. Li, S. Zhang, and X. Li, "Rupture degree of graphs," International Journal of Computer Mathematics, vol. 82, no. 7, pp. 793803, 2005.

[5] S. S. Y. Wu and M. B. Cozzens, "The minimum size of critically m-neighbour-connected graphs," Ars Combinatoria, vol. 29, pp. 149-160, 1990.

[6] G. Gunther, "Neighbour-connectivity in regular graphs," Discrete Applied Mathematics, vol. 11, no. 3, pp. 233-243, 1985.

[7] M. B. Cozzens and S. S. Y. Wu, "Extreme values of the edgeneighbor-connectivity," Ars Combinatoria, vol. 39, pp. 199-210, 1995.

[8] M. B. Cozzens and S. S.Y. Wu, "Vertex-neighbor-integrity of trees," Ars Combinatoria, vol. 43, pp. 169-180, 1996.

[9] M. B. Cozzens and S. S. Y. Wu, "Edge-neighbor-integrity of trees," The Australasian Journal of Combinatorics, vol. 10, pp. 163-174, 1994.

[10] Z. Wei, A. Mai, and M. Zhai, "Vertex-neighbor-scattering number of graphs," Ars Combinatoria, vol. 102, pp. 417-426, 2011.

[11] Z. Wei, Y. Li, and J. Zhang, "Edge-neighbor-scattering number of graphs," Ars Combinatoria, vol. 85, pp. 271-277, 2007.

[12] G. Bacak-Turan and A. Kırlangıç, "Neighbor rupture degree and the relations between other parameters," Ars Combinatoria, vol. 102, pp. 333-352, 2011.

[13] M. B. Cozzens and S. S.Y. Wu, "Vertex-neighbor-integrity of powers of cycles," Ars Combinatoria, vol. 48, pp. 257-270, 1998. 


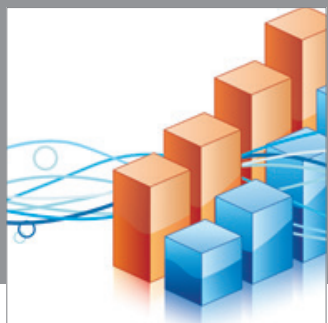

Advances in

Operations Research

mansans

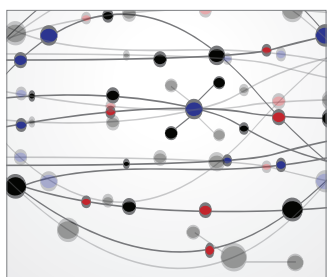

The Scientific World Journal
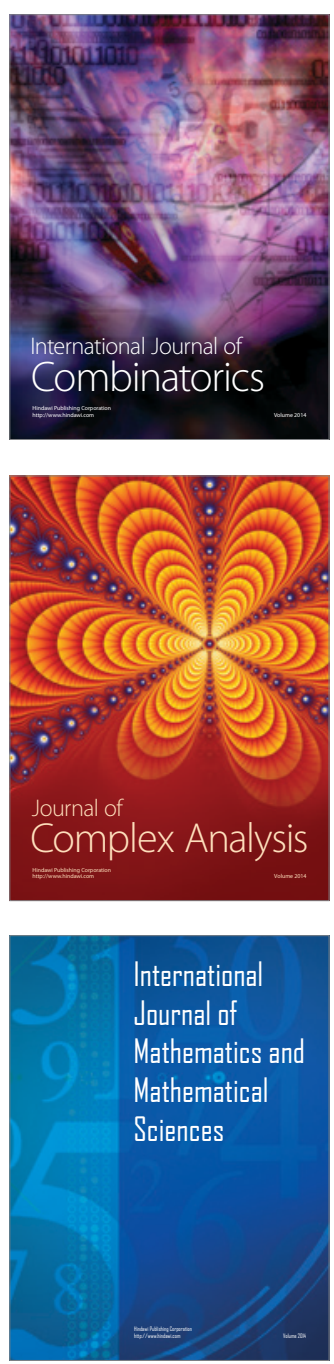
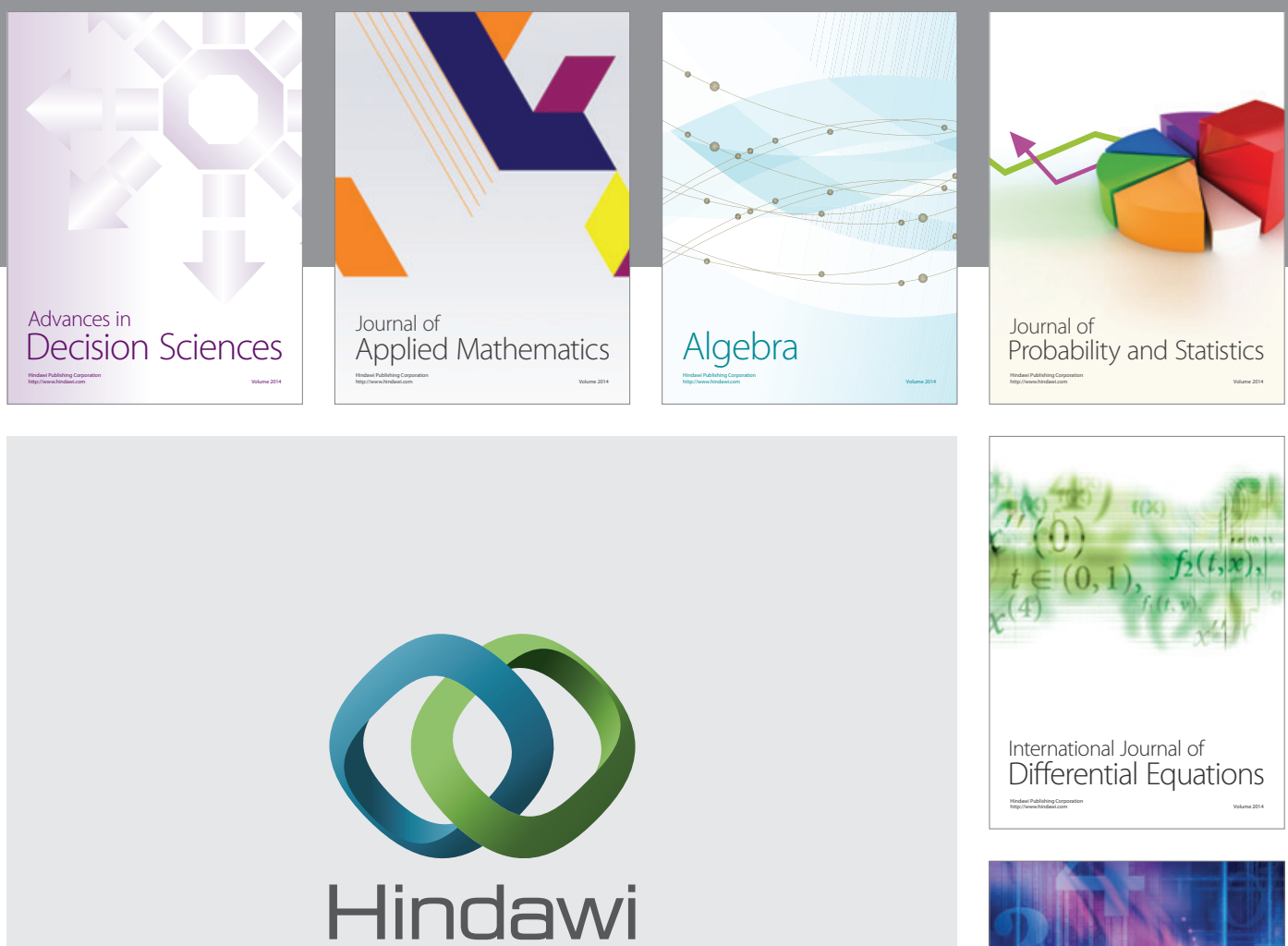

Submit your manuscripts at http://www.hindawi.com
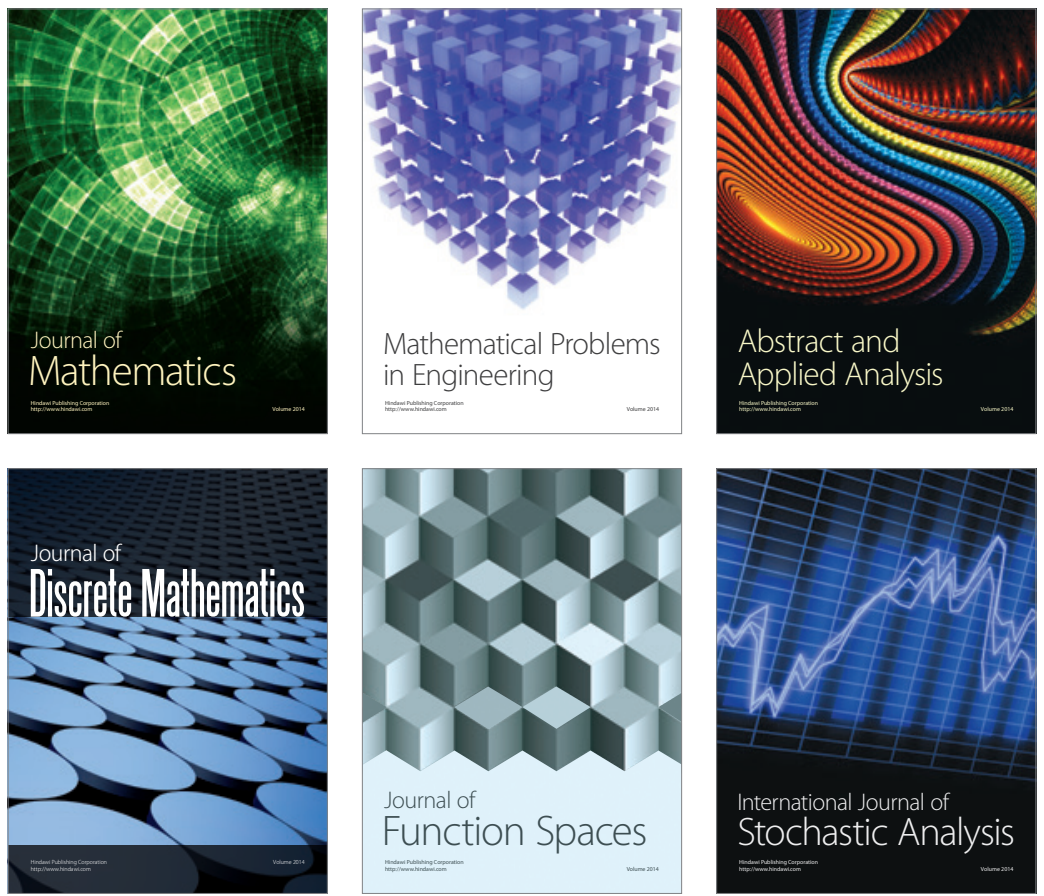

Journal of

Function Spaces

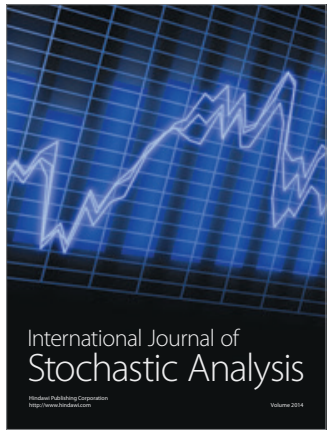

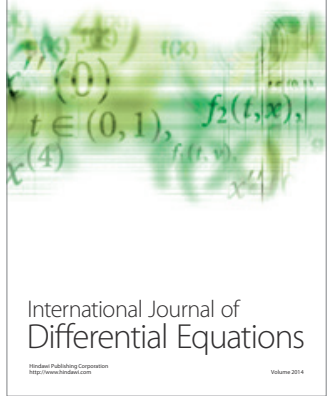
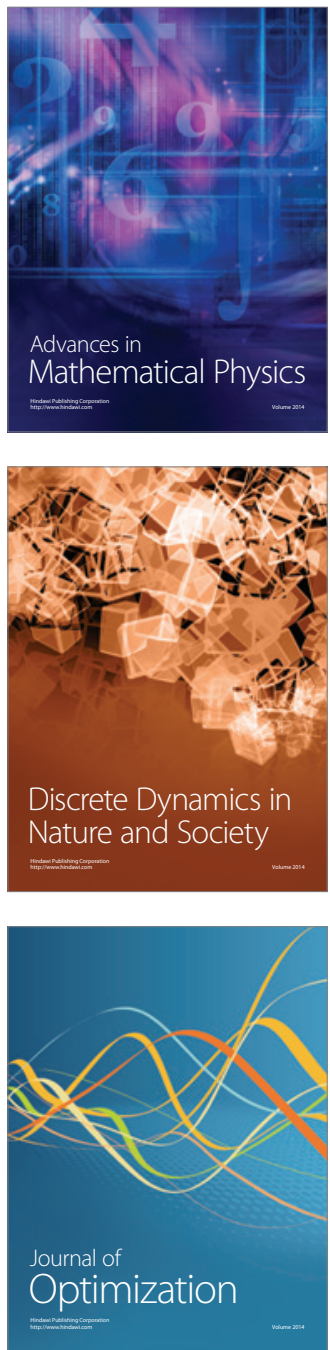